\title{
Molecular Characterization and Pathology of Virulent Newcastle Disease Virus Isolated from Broiler Chickens in District Lahore, Pakistan
}

M. Ali', M.U.R. Khan ${ }^{1}$, A. Aslam¹, H.U. Rehman², A. Anjum³,

S.F. Waheed ${ }^{1}$, S. Usman' 1 , S. Masood ${ }^{4}$, A. Ullah ${ }^{5}$, A. Masood ${ }^{6}$

10.18805/IJAR.BF-1420

\begin{abstract}
Background: This study elucidated the molecular detection and pathological alterations in broiler chickens naturally infected with field circulating NDV strains along with their phylogenomic dynamics.

Methods: Morbid tissue samples of diseased/dead chickens were collected from 100 poultry flocks presented to poultry disease diagnostic laboratories from September 2018 to August 2019. Samples were subjected to molecular detection of NDV along with phylogenetic analysis and subsequent gross and histopathological examination.

Result: Based on RT-PCR results, the positivity of NDV was 04/100 (4\%). Genetic analysis of the NDV Fusion (F) gene revealed $98.92 \%$ and $98.74 \%$ similarity with Iranian and Pakistani isolates, respectively. The evolutionary tree showed that present study isolates were placed in a clade belongs to genotype VII sub-genotype $i$ and I. Necropsy examinations revealed the petechial haemorrhages associated with multifocal necrosis in gastrointestinal and respiratory organs. Besides these pathological findings, amino acid sequence of $\mathrm{F}$ gene revealed that study isolates are having pathogenic potential similar to the velogenic strains of NDV. Based on all essential analyses, the present study concluded that the evolution and distribution of the Newcastle disease virus of various genotypes VIIi and VIII in Pakistan are having significant pathogenic potential. Therefore, it emphasizes developing ND vaccine from indigenous strains for better protection of commercial poultry in Pakistan.
\end{abstract}

Key words: Chicken, Histopathology, Pathology, Phylogenetic analysis, Polymerase chain reaction.

\section{INTRODUCTION}

Among the largest poultry producers in the world, Pakistan is now placed at the 11 th position with its poultry sector growing at an impressive rate of approximately 7.5 per cent per annum over the last decade. Chicken meat is not only a cheaper source of quality nutrition; it has also the therapeutic potential due to higher antioxidant property of chicken with black meat (Sehrawat et al., 2021). Economics of poultry production can be improved by reducing input on health care Jaiswal et al. (2014). Among the infectious diseases, RNA viruses remain potential threat for commercial poultry due to virus evolving nature. During 2006 and 2009, Newcastle disease (ND) was ranked as the $3^{\text {rd }}$ most crucial poultry disease (Rehan et al., 2019). Since the development of an organized poultry sector in Pakistan, NDV has been devastating to the poultry industry for many years. Domestic poultry is highly susceptible to ND infection, responsible for severe outbreaks worldwide, resulting in million-dollar losses to farmers (Rahman et al. 2016). The Newcastle disease virus (NDV) or Avian orthoavulavirus 1 causes Newcastle disease, which is an economically important transboundary viral disease. Virulent strains of NDV are highly contagious and can be devastating, especially in immune-compromised chicken (Ganar et al. 2014).

Despite widespread vaccination and stringent biosecurity steps, ND outbreaks are also reported in vaccinated flocks (Etriwati et al. 2017) while its incidence is
'Department of Pathology, University of Veterinary and Animal Sciences, Lahore-54000, Pakistan.

${ }^{2}$ Department of Physiology, University of Veterinary and Animal Sciences, Lahore-54000, Pakistan.

${ }^{3}$ Department of Pathology, The Islamia University of Bahawalpur63100, Pakistan.

${ }^{4}$ Department of Anatomy and Histology, University of Veterinary and Animal Sciences, Lahore-54000, Pakistan.

${ }^{5}$ Institute of Biochemistry and Biotechnology, University of Veterinary and Animal Sciences, Lahore-54000, Pakistan.

${ }^{6}$ Department of Anatomy and Histology, The Islamia University of Bahawalpur-63100, Pakistan.

Corresponding Author: M.U.R. Khan, Department of Pathology, University of Veterinary and Animal Sciences, Lahore-54000, Pakistan. Email: drniazi@uvas.edu.pk

How to cite this article: Ali, M., Khan, M.U.R., Aslam, A., Rehman, H.U., Anjum, A., Waheed, S.F., Usman, S., Masood, S., Ullah, A. and Masood, A. (2021). Molecular Characterization and Pathology of Virulent Newcastle Disease Virus Isolated from Broiler Chickens in District Lahore, Pakistan. Indian Journal of Animal Research. DOI: $10.18805 /$ IJAR.BF-1420.

Submitted: 09-08-2021 Accepted: 08-10-2021 Online: 18-11-2021

higher in non-vaccinated flocks. However, outbreaks in recent years have raised concerns regarding vaccination failure, which may occur due to incompatibility between field and vaccine strains or immunosuppressed birds which do 
not produce NDV protective titer (Mehmood et al. 2019). The broad host range of NDV in avian species has contributed to a substantial genetic variation of the virus and the emergence of NDV variants. In this respect, the isolation of novel NDV variants with different pathogenicity from diversified hosts indicates the virus evolution with subsequent disease transmission implications (Dimitrov et al. 2016). The phylogenomic studies found that 21 genotypes circulate in multiple avian species globally, with genotypes VII, XIII and XXI type of viruses were causing most outbreaks in developing countries including Pakistan (Rahman et al. 2020b). A reference index for AAvV pathotyping can be established through intracerebral pathogenicity index in one-day-old chickens, disease severity in susceptible hosts and analysis of fusion protein cleavage motifs (Rahman et al. 2019a). Very virulent NDV isolates have at least three basic amino acids i.e. arginine $(\mathrm{R})$ or lysine $(\mathrm{K})$ starting at positions 112-116 with phenylalanine $(F)$ at position 117 , while low virulent viruses have basic amino acids only at positions 113 and 116 with leucine (L) at position 117 (Alexander et al. (2012).

Due to frequent outbreaks of ND in commercial chicken flocks, poultry farmers have to bear heavy economic losses every year. Lahore is the second largest city of Pakistan and is located at $31.52^{\circ} \mathrm{N} 74.35^{\circ} \mathrm{E}$. Established poultry disease diagnostic centers by the public and private sectors in Lahore were approached for convenient sampling. This study was designed to identify the current field circulating NDV based on F gene from diseased and sick chicken flocks. Furthermore, phylogenomic analysis was conducted to give an insight towards the genetic diversity of circulating indigenous strains and comparative analysis with the previously described strains. Pathology of field circulating NDV was recorded by monitoring gross and histopathological changes in the morbid tissues.

\section{MATERIALS AND METHODS}

From September 2018 to August 2019, morbid tissue samples (trachea, lungs, intestine) of NDV vaccinated commercial chickens were aseptically collected and pooled from different broiler poultry farms $(n=100)$ of the Lahore district (Pakistan). About 3-5 birds' samples were collected from a single flock. After observing the pathological lesions, the collected samples were adequately labelled and divided into two parts; for reverse transcriptase-polymerase chain reaction (RT-PCR), samples were placed in an icebox and for histopathology, samples were preserved into $10 \%$ neutral buffered formalin. The whole laboratory work was done in molecular pathology laboratory and histopathology laboratory at Department of Pathology, University of Veterinary and Animal Sciences Lahore.

For molecular detection of NDV, tracheal tissue from each 3-5 birds of every flock was pooled and triturated in sterile phosphate buffer saline (PBS). Tissue homogenates were centrifuged at 5,000 $\mathrm{g}$ for $3 \mathrm{~min}$ and the supernatants were inoculated $(0.2 \mathrm{ml})$ in a 9 -day old chicken embryonated eggs with no maternal antibodies against ND. Harvested allantoic fluid was subjected to spot haemagglutination assay and then haemagglutination inhibition assay using known hyperimmune serum for NDV. The positive samples were subjected to RNA extraction, cDNA synthesis and RT-PCR as described by Saad et al. (2021). Previously reported primer set (NDV-F 589 5'-GGAGGATGTTGGCAGCATT-3' and NDV-R 575S 5'- GTCAACATATACACCTCATC-3') were used to amplify NDV fusion gene fragment (589 bp) Pang et al. (2002). RT-PCR amplification was conducted in a final volume of $25 \mu \mathrm{l}$, containing PCR master mix (2X, Thermo Scientific, United States) $(12.5 \mu \mathrm{l})$, DNA template $(2 \mu \mathrm{l})$, primer forward and reverse $(1.5 \mu$ l, each) and PCR grade water $(7.5 \mu \mathrm{l})$. A Veriti TM 96-Well Thermal Cycler (Applied Biosystems ${ }^{\mathrm{TM}}$, United States) was adjusted to $95^{\circ} \mathrm{C}$ for 2 min (pre-denaturation) followed by 35 cycles of denaturation $\left(95^{\circ} \mathrm{C}\right.$ for $\left.10 \mathrm{sec}\right)$, annealing $\left(53^{\circ} \mathrm{C}\right.$ for $\left.30 \mathrm{sec}\right)$ and extension $\left(72^{\circ} \mathrm{C}\right.$ for $\left.45 \mathrm{sec}\right)$ with a final incubation of $72^{\circ} \mathrm{C}$ for $5 \mathrm{~min}$. The PCR product was analyzed by gel electrophoresis against a 100 bp DNA ladder (Thermo Scientific, United States).

The purified PCR products in four positive samples for NDV were subjected to the Sanger Sequencing method. The nucleotide sequences were placed in a public database (http://www.ncbi.nlm.nih.gov/GenBank accessed on July 2, $2021)$ to retrieve the genome sequences $(n=66)$ of avian host Newcastle disease virus reported in different geographic regions worldwide. The obtained nucleotide and deduced amino acid sequences were aligned and analyzed through ClustalW on BioEdit version 7.2.5. The output file was subjected to a distance-based neighbour-joining model in MEGA-X software to construct the evolutionary tree. The reliability of the tree was based on 1,000 bootstrap replicates. Gross pathological changes were recorded from morbid tissues collected from sick birds found positive for NDV by PCR. While formalin-fixed tissue samples were processed for histopathology and sections of $4 \mu \mathrm{m}$ thickness were stained with routine hematoxylin and eosin (H\&E stain) to get examined under a light microscope for the recording of microscopic changes Śliżewska et al. (2019).

\section{RESULTS AND DISCUSSION}

\section{Molecular identification and genetic characterization of NDV}

Poultry production remains under challenge by infectious diseases. Despite vigorous vaccination, Newcastle disease (ND) is one of the prevalent diseases in Pakistan causing economic losses to the farmers. NDV F gene is well recognized genetic marker and capable of generating evolutionary information. $\mathrm{F}$ gene in NDV requires activation by proteolytic cleavage for infectivity. OIE categories NDV as velogenic when multiple basic amino acid sequences are present at cleavage sites of position 112-116 and phenylalanine $(F)$ at position 117 in the $F$ gene amino acid chain (OIE, 2012). Based on RT-PCR results, 04/100 (4\%) samples were found positive for NDV by amplifying the 
product size 589bp (Fig 1). Due to high genome sequence similarities, they were grouped into two isolates. The partial gene sequences of the present study were submitted to the NCBI-GenBank database under accession numbers MW295940 (Avian avulavirus 1 isolate chicken/Pakistan/ PATH-IV/2019) and MW427214 (Avian avulavirus 1 isolate chicken/Pakistan/PATH-VI/2018). In this study, NDV isolates showed the presence of multibasic amino acid cleavage sequence (RRQKRF) in F gene (Fig 4).

The phylogenetic analysis represented that all retrieved isolates were clustered into two classes i.e., class 1 (avirulent in chicken) and class II (virulent in chicken). While NDVclass II was categorized into 21 genotypes. The pairwise homology distance matrix analysis revealed that our isolates

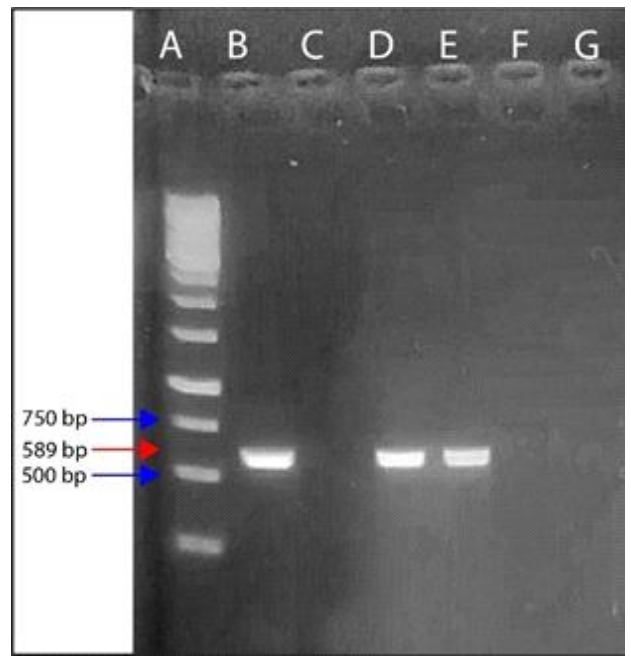

Fig 1: Agarose gel electrophoresis of Newcastle disease virus. Lane A: DNA ladder 1kb plus, Lane B: positive control, Lane C: negative control. Lane D, E: positive samples (589bp amplicon size). Lane F, G: negative samples. had a maximum percent identity matrix (nucleotide: $91.33 \%$ to $99.49 \%$ and amino acid: $96.43 \%$ to $100.00 \%$ ) with NDV genotype VII (Table 1).

Current study isolates were grouped within genotype VII where most of the recent Pakistani NDV isolates have been reported (Fig 2). Percentage nucleotide similarity showed that one isolate has maximum similarity with Iranian isolate (MH481362) of genotype VII and sub-genotype I which is not earlier reported from Pakistan. While second NDV isolate of the current study showed maximum similarity with already NDV isolate reported from Pakistan in 2018 belonging to genotype VII and sub-genotype i (Fig 3). In Pakistan, NDV genotype VII, XIII and XXI are primarily reported in a wide range of hosts. Apart from this, genotype VII is crucial because of its prevalence in the clinically healthy backyard and asymptomatic wild waterfowl Rahman et al. (2019b). This highlights the possibility of introducing this strain through the transboundary trade of poultry flocks as well as a possible way of NDV spread through migratory birds.

\section{Gross and histopathological examination}

The clinical sign observed in the birds representing NDV affected flock were greenish diarrhea, depression, anorexia, discharge from the oral cavity, respiratory discomfort, oculonasal discharge, torticollis and up to $40 \%$ mortality. Similar clinical signs were recorded in an experimental velogenic NDV infection to chicken by Rahman et al. (2019c). Among the gross lesion observed, congestion and oedematous lungs were noted in NDV affected birds (Fig 5b). Trachea showing the scattered haemorrhages with exudate in lumen were most predominant macroscopic lesions in the respiratory tract ( $($ Fig $5 c)$ which are consistent with earlier reports in chicken with velogenic viscerotropic NDV infection Brar et al. (2017). Pinpoint haemorrhages were found on

Table 1: Detailed information of pairwise distance identity matrix analysis of understudy isolates.

\begin{tabular}{|c|c|c|c|c|c|c|c|c|c|}
\hline \multirow{2}{*}{$\begin{array}{l}\text { Accession } \\
\text { numbers }\end{array}$} & \multirow[t]{2}{*}{ Strains/isolates } & \multirow[t]{2}{*}{ Genotype } & \multirow{2}{*}{$\begin{array}{c}\text { Year } \\
\text { of } \\
\text { isolation }\end{array}$} & \multirow[t]{2}{*}{ Host } & \multirow[t]{2}{*}{ Country } & \multicolumn{2}{|c|}{$\begin{array}{l}\text { MW295940 } \\
\text { \%age identity }\end{array}$} & \multicolumn{2}{|c|}{$\begin{array}{l}\text { MW427214 } \\
\text { \%age identity }\end{array}$} \\
\hline & & & & & & Nucleotide & Amino acid & Nucleotide & Amino acid \\
\hline MN481197 & Ck/IR/EMA132/2018 & VII-I & 2018 & Chicken & Iran & $99.49 \%$ & $100.00 \%$ & $91.33 \%$ & $96.43 \%$ \\
\hline MG686600 & Duck/NDV/Pak & VII-i & 2015 & Duck & Pakistan & $91.67 \%$ & $96.94 \%$ & $99.83 \%$ & $99.49 \%$ \\
\hline KY042135 & pigeon/Pakistan/2015 & $\mathrm{XXI}$ & 2015 & Pigeon & Pakistan & $89.46 \%$ & $94.39 \%$ & $88.10 \%$ & $93.88 \%$ \\
\hline FJ766526 & $\mathrm{JS} / 07 / 22 / \mathrm{Pi}$ & VI & 2007 & Pigeon & China & $89.46 \%$ & $94.90 \%$ & $88.78 \%$ & $95.41 \%$ \\
\hline KU862298 & Pigeon/Pak/2015 & $X X I$ & 2015 & Pigeon & Pakistan & $88.95 \%$ & $93.88 \%$ & $88.10 \%$ & $92.86 \%$ \\
\hline MH019283 & PK3 & XIII & 2015 & Chicken & Pakistan & $88.44 \%$ & $94.90 \%$ & $88.95 \%$ & $96.43 \%$ \\
\hline KP027403 & Layer/China/Yulin/10 & $\mathrm{IX}$ & 2010 & Layer & China & $87.59 \%$ & $92.35 \%$ & $88.10 \%$ & $94.39 \%$ \\
\hline MN393176 & NDV-F8 & VIII & 1998 & Avian & Vietnam & $87.41 \%$ & $94.39 \%$ & $88.27 \%$ & $96.43 \%$ \\
\hline AY562987 & gamefowl/U.S./02 & V & 2002 & Game fowl & USA & $86.73 \%$ & $91.84 \%$ & $85.37 \%$ & $92.86 \%$ \\
\hline EF201805 & Mukteswar & III & 2007 & Avian & China & $85.71 \%$ & $91.33 \%$ & $87.07 \%$ & $92.35 \%$ \\
\hline AY935500 & I-2progenitor & 1 & 2006 & Avian & Australia & $84.18 \%$ & $89.80 \%$ & $87.07 \%$ & $91.84 \%$ \\
\hline AF077761 & LaSota & II & 1998 & Avian & Netherland & $83.67 \%$ & $88.78 \%$ & $84.18 \%$ & $90.82 \%$ \\
\hline KX857718 & duck/USA/2009 & $x$ & 2009 & black duck & USA & $83.67 \%$ & $89.80 \%$ & $84.52 \%$ & $91.84 \%$ \\
\hline HQ266602 & MG_725_08 & $X I$ & 2008 & Chicken & Madagscar & $83.67 \%$ & $87.76 \%$ & $83.16 \%$ & $89.80 \%$ \\
\hline FJ794269 & NDV08-004 & Class I & 2008 & Duck & China & $71.43 \%$ & $85.20 \%$ & $70.41 \%$ & $86.22 \%$ \\
\hline
\end{tabular}


the mucosal tips of proventriculus papillae (Fig 5e). In the intestine wall, clearly demarcated button-like hemorrhagic ulcers were present (Fig 5a). The pathologic changes observed in the proventriculus and small intestine are the pathognomonic lesions of velogenic viscerotropic NDV strain Fentie et al. (2014). Hemorrhagic caecal tonsils were present in the NDV positive birds (Fig $5 \mathrm{~d}$ ). The gross pathology in the present study suggested that the extent of lesions was more severe in the proventriculus, intestine, trachea, lungs and caecal tonsils which were also recorded in previous studies (Etriwati et al., 2017; Nakamura et al., 2014; Rahman et al., 2019a). In the histopathological examination of morbid tissues ((Fig 6), tracheal tissue showed degeneration and necrosis of mucosal epithelium along with congested

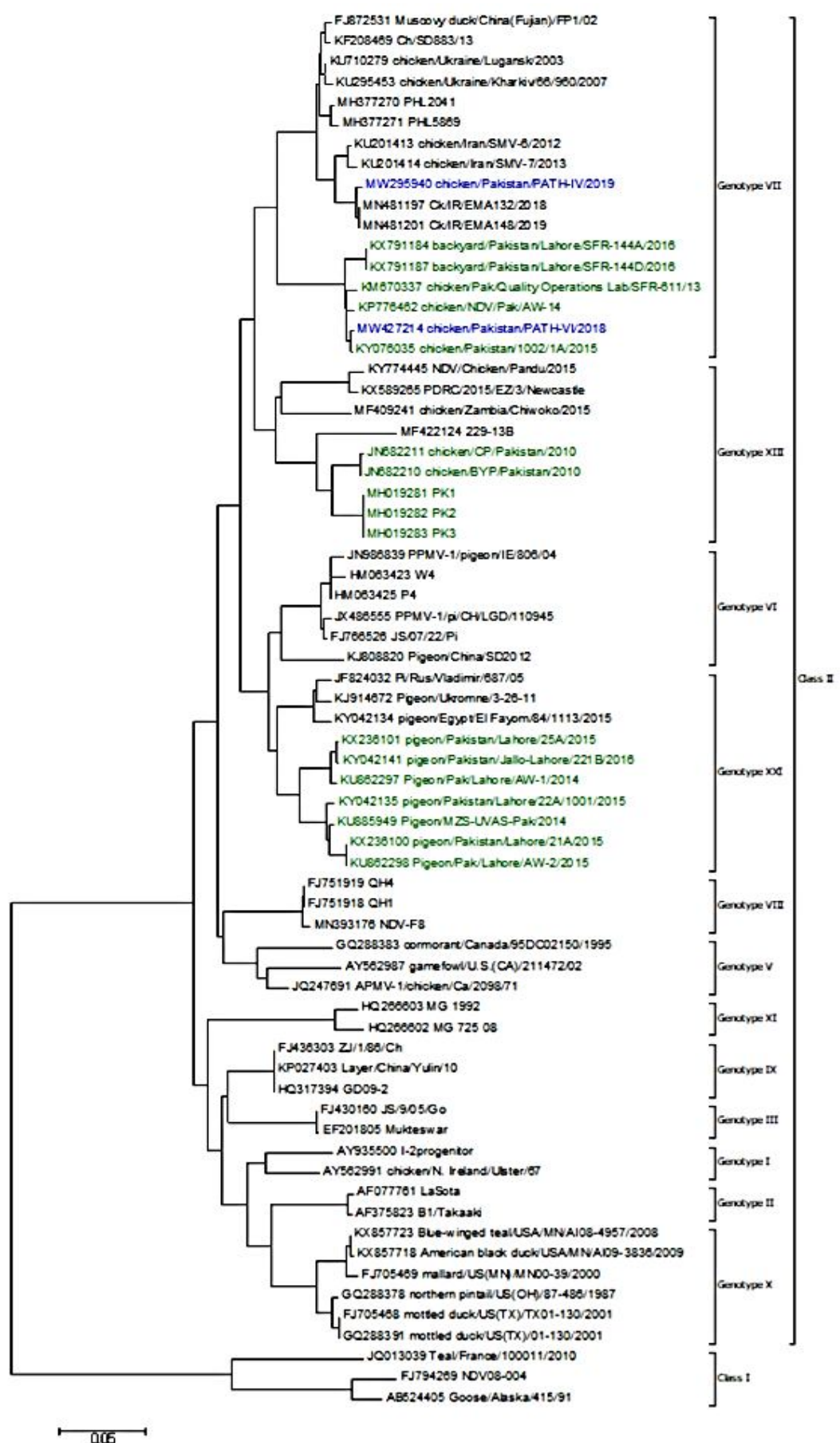

Fig 2: Evolutionary tree constructed based on F-gene sequences of NDV. Green highlighted isolates belong to Pakistan, while highlighted with blue represent present study isolates. 


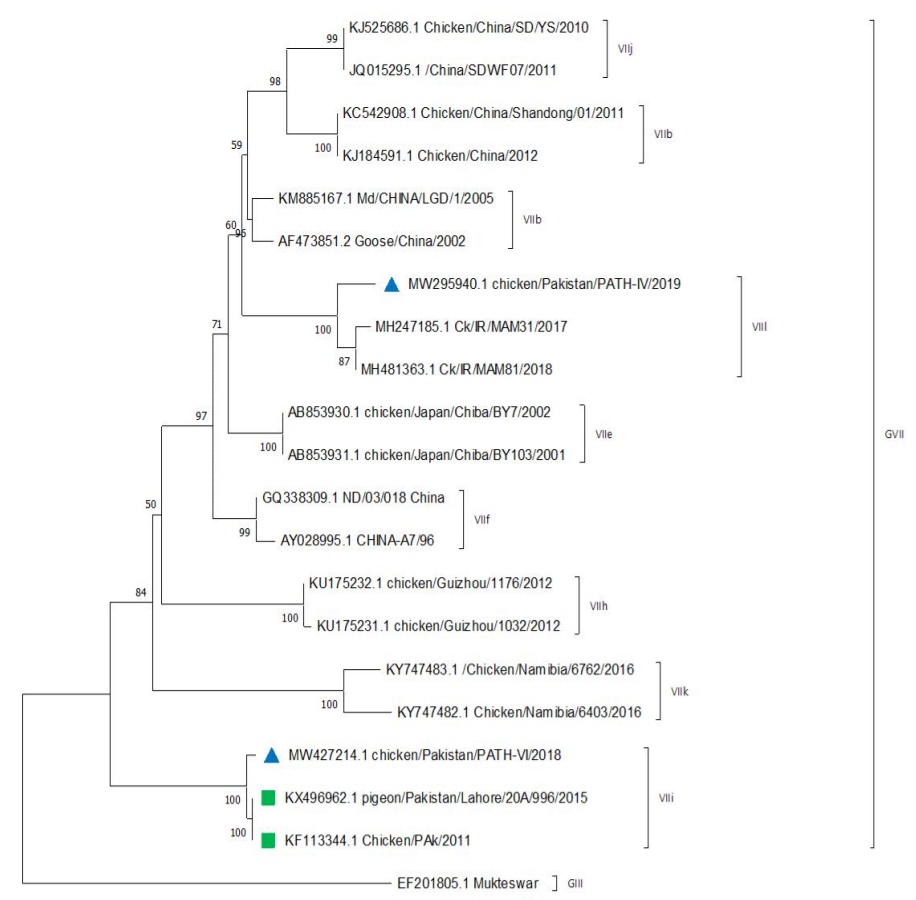

0.02

Fig 3: Evolutionary tree constructed based on F-gene sequences of NDV genotype VII reported in different geographical regions of the world. Blue triangle marked highlighted isolates present study isolates (G VIli and G VIII) and green box showing Pakistani NDV isolates (G VIli).

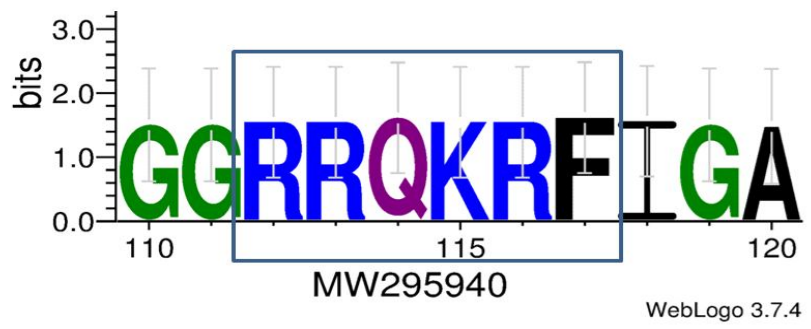

Fig 4: F protein multibasic amino acid cleavage sequence from current study NDV isolate (MW295940) in blue square showing virulent NDV (analyzed by WebLogo 3.1).

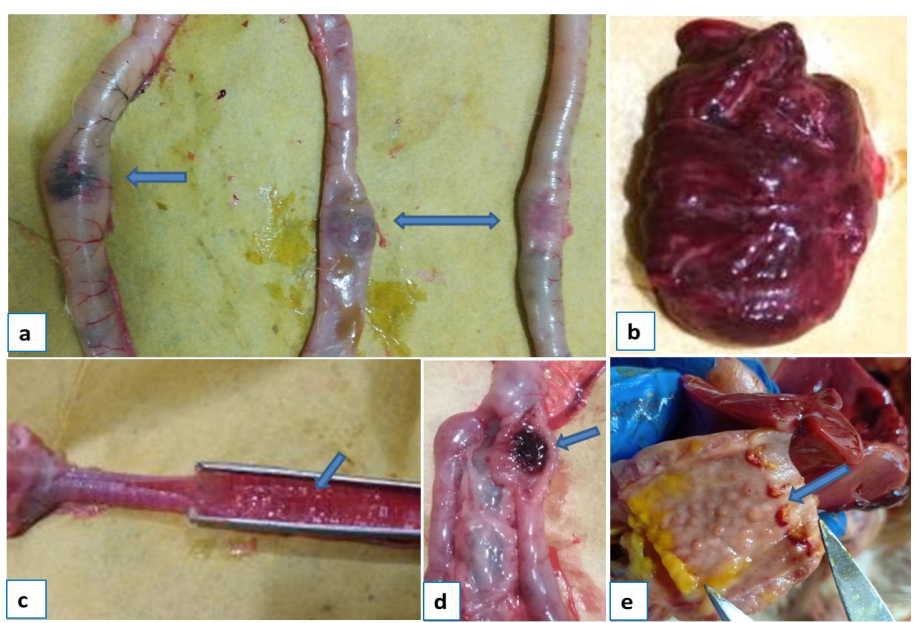

Fig 5: Photographs of NDV infected birds morbid tissues (a) necrotic button like ulcer on Jejunum (arrow) (b) pulmonary oedema and congestion (c) congested tracheal mucosa with exudate (arrow) (d) inflamed and hemorrhagic caecal tonsils (arrow) (e) ecchymotic hemorrhages on proventiculus mucosal glands tips (arrow). 
capillaries. In the lung parenchyma, there were infiltration of mononuclear cells were observed along with congestion in the lumen of parabronchi. Intestinal tissue showed sloughed villi epithelium and marked infiltration of lymphohistiocytes in the mucosa. Brar et al. (2017) also reported the following lesions of velogenic NDV infected chickens; degenerative changes in tracheal epithelial cells, pulmonary congestion and sloughing of intestinal villi.

ND live vaccines being currently used in Pakistan belongs to G-1I (Lasota) and G-III (Mukhteswar). However, there are reports of morbidity and mortality even in vaccinated poultry flocks (Etriwati et al., 2017; Mehmood

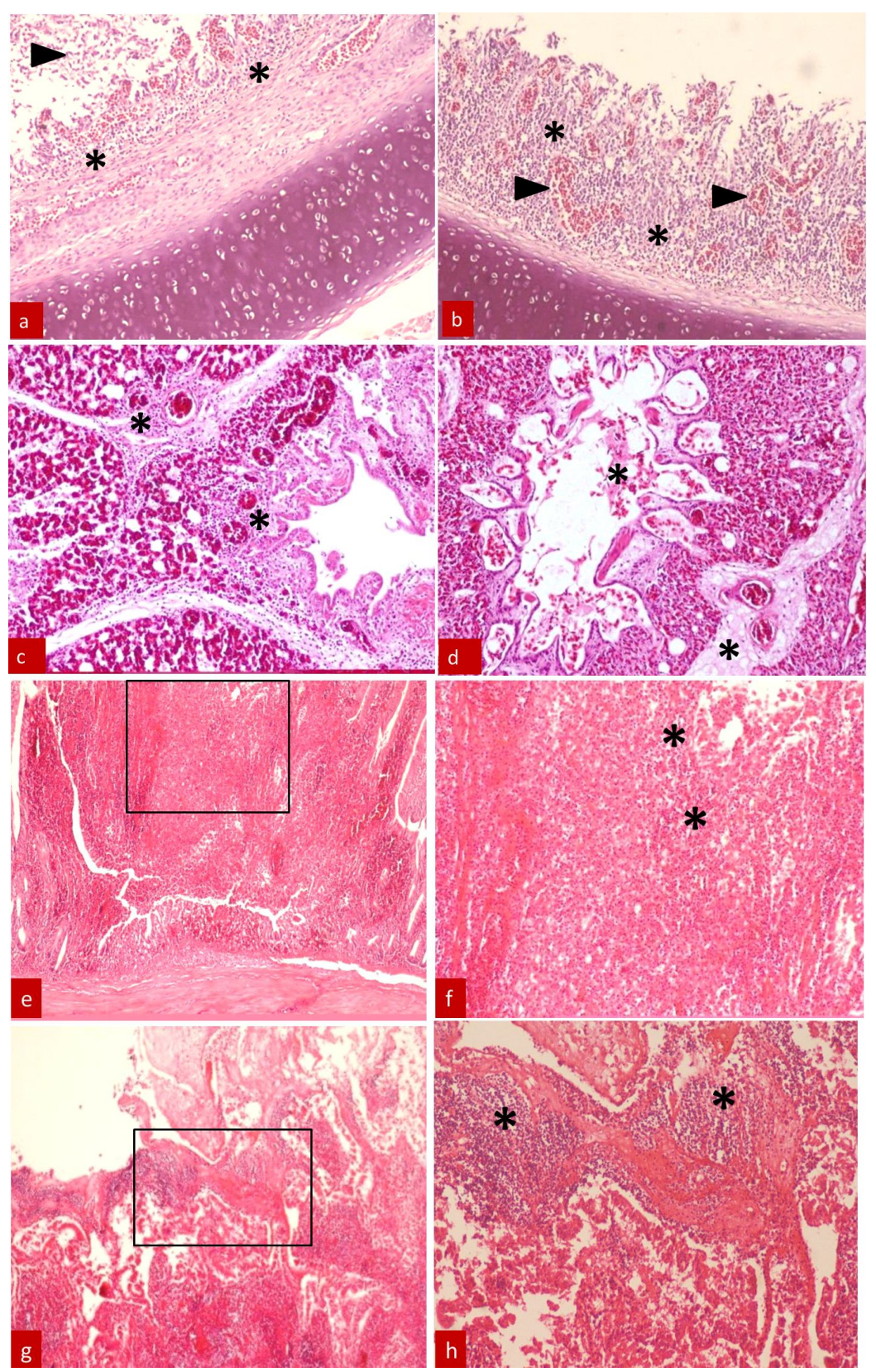

Fig 6: (a). Accumulation of necrotic cellular debris in tracheal lumen (arrow head), lymphoplasmacytic infiltrate in tracheal mucosa (asterik) with complete loss of cilia. 10x (b). Iymphoplasmacytic infiltrate in tracheal mucosa (asterik), degeneration of mucosal epithelia cells, engorged capillaries (arrow head). 10x (c). Marked lymphoplasmacytic infiltration in the parabronchial wall and interstitium (asterik). 10x (d). fibrinoheterophilic exudate present in parabronchial lumen. 10x (e). Jejunum; lamina propria is expanded with infiltration of mononeuclear inflammatory cells (marked with box) $4 x(f)$. Jejunum. Higher power view of area in "e" showing Infiltration of mononuclear inflammatory cells (asterik). 10x (g). Jejunum. erosion and distortion of mucosal surface of villi, marked lymphohistiocytic infiltration in mucosa and submucosa $4 x(h)$. Jejunum. Higher power view of area in " $g$ " Necrotic debris showing marked Lymphohistiocytic infiltration (asterik). 10x. 
et al., 2019; Perozo et al., 2012). Rehmani et al. (2015) has found virulent NDV genotype VIli in vaccinated chicken in Pakistan. In the present study, NDV of G-VIli and G-VIII in vaccinated flocks with a high degree of morbidity and mortality imparts significance to the development of ND vaccine from indigenous isolate for better immunity and regular monitoring of antibody titer so the virulent virus cannot hit the flock with low immunity against the diseases. It can be drawn from the study results that NDV killed vaccine belonging to genotype VII prepared from indigenous isolate should be included in vaccination regime for protection against the current circulating NDV belonging to G-VII.

\section{CONCLUSION}

It is the first to report NDV G VIII isolated from broiler chicken in Pakistan to the best of our knowledge. The current study demonstrated the molecular characteristics and pathology of field circulating NDV. Based on the gross lesion, histopathological examination and amino acid sequence of $\mathrm{F}$ gene, it is elucidated that isolated NDV has the pathogenic potential of velogenic NDV strain. ND in vaccinated flocks with a high degree of morbidity and mortality imparts significance to develop ND vaccine from indigenous isolate for better immunity and strict biosecurity measures be adopted which otherwise can breach the bird's immunity against the diseases. Robust research and development activities are still warranted to save huge losses in the poultry industry of Pakistan. Therefore, in disease-endemic countries, continuous surveillance of viral evolution should be ensured for disease control interventions.

\section{ACKNOWLEDGEMENT}

This study was financially supported by the Higher Education Commission of Pakistan through PhD Indigenous Scholarships Scheme (PIN No.518-1084212AV5-101).

\section{REFERENCES}

Alexander, D.J., Aldous, E.W., Fuller, C.M. (2012). The long view: a selective review of 40 years of Newcastle disease research. Avian Pathology. 41: 329-335.

Brar, R., Leishangthem, G.D., Gadhave, P., Singh, N., Banga, H., Mahajan, V., Sodhi, S. (2017). Diagnosis of Newcastle disease in broiler by histopathology and immunohistochemistry. Indian Journal of Veterinary Pathology. 41: 60-62.

Dimitrov, K.M., Ramey, A.M., Quu, X., Bahl, J. and Afonso, C.L. (2016). Temporal,geographic and host distribution of avian paramyxovirus 1 (Newcastle disease virus). Infection, Genetics and Evolution. 39: 22-34.

Etriwati, R.D., Handharyani, E., Setiyaningsih, S. (2017). Pathology and immunohistochemistry study of Newcastle disease field case in chicken in Indonesia. Veterinary World. 10: 1066-1071.
Fentie, T., Dadi, K., Kassa, T., Sahle, M., Cattoli, G. (2014). Effect of vaccination on transmission characteristics of highly virulent Newcastle disease virus in experimentally infected chickens. Avian Pathology. 43: 420-426.

Ganar, K., Das, M., Sinha, S., Kumar, S. (2014). Newcastle disease virus: Current status and our understanding. Virus Research. 184: 71-81.

Jaiswal, G., Kumar, S., Prasad, Y. (2014). Immunocompetence traits and their inheritance pattern in kadaknath native chicken. Indian Journal of Animal Research. 48: 509-512.

Mehmood, A., Rasool, M.H., Anjum, A.A., Zahoor, M.A., Shafique, M. (2019). Pathotyping and genetic characterization of a highly virulent Newcastle disease virus strain isolated from recent outbreak in District Okara, Pakistan. Pakistan Veterinary Journal. 39: 353-358.

Nakamura, K., Ito, M., Nakamura, T., Yamamoto, Y., Yamada, M., Mase, M., Imai, K. (2014). Pathogenesis of Newcastle disease in vaccinated chickens: Pathogenicity of isolated virus and vaccine effect on challenge of its virus. Journal of Veterinary Medical Science. 76: 31-36.

OIE. (2012). Newcastle Disease. In Manual of Diagnostic Tests and Vaccines for Terrestrial Animals: Mammals, Birds and Bees; Biological Standards Commission: Paris, France. 1: 555-574.

Pang, Y., Wang, H., Girshick, T., Xie, Z., Khan, M.I. (2002). Development and application of a multiplex polymerase chain reaction for avian respiratory agents. Avian Diseases. 46: 691-699.

Perozo, F., Marcano, R., Afonso, C.L. (2012). Biological and phylogenetic characterization of a genotype VII Newcastle disease virus from Venezuela: efficacy of field vaccination. Journal of Clinical Microbiology. 50: 1204-8.

Rahman, A., Habib, M., Riaz, T., Hussain, B., Yousaf, F., Saqalein, M., Rasool, M.H. (2016). Seroprevalence of Newcastle disease virus (NDV) in commercial and domesticated birds: During current surge of NDV in Pakistan. Journal of Infection and Molecular Biology. 4: 54-59.

Rahman, A., Munir, M., Shabbir, M.Z. (2019a). A comparative genomic and evolutionary analysis of circulating strains of Avian avulavirus 1 in Pakistan. Molecular Genetics and Genomics. 294: 1289-1309.

Rahman, A. and Shabbir, M.Z. (2019b). A comparative phylogenomic analysis of Avian avulavirus 1 isolated from non-avian hosts: Conquering new frontiers of zoonotic potential among species. Archives of Virology. 164: 1771-1780.

Rahman, A., Rohaim, M.A., Rania, F., Naggar, E., Mustafa, G., Chaudhry, U., Shabbir, M.Z., (2019c). Comparative clinico-pathological assessment of velogenic (sub-genotype VIli) and mesogenic (sub-genotype VIm) Avian avulavirus 1 in chickens and pigeons, Avian Pathology. 48: 610-621.

Rahman, A., Shabbir, M.A.B., Mehmood, A., Shabbir, M.Z. (2020b). Genotypic and subgenotypic diversity of avian paramyxo viruses 2, 4 and 6. Pakistan Veterinary Journal. 40: $1-4$.

Rehan, M., Aslam, A., Khan, M., Abid, M., Hussain, S., Amber, J., Anjum, A., Hussain, A. (2019). Potential economic impact of Newcastle disease virus isolated from wild birds on commercial poultry industry of Pakistan: A review. Hosts Viruses. 6: 1-15. 
Molecular Characterization and Pathology of Virulent Newcastle Disease Virus Isolated from Broiler Chickens in District Lahore, Pakistan

Rehmani, S.F., Wajid, A., Bibi, T., Nazir, B., Mukhtar, N., Hussain, A., Lone, N.A., Yaqub, T., Afonso, C.L. (2015). Presence of virulent newcastle disease virus in vaccinated chickens in farms in Pakistan. Journal of Clinical Microbiology. 53: 1715-1718.

Saad, K., Elfatah, A., Abas Elabasy, M., El-Khyate, F., Kotb Elmahallawy, E., Mosad, S.M., El-Gohary, F.A., Abdo, W., Al-Brakati, A., Seadawy, M.G., Tahoon, A.E., Elgalil ElGohary, A., Lupini, C., Mescolini, G., Eg, A.E.E. (2021). Molecular characterization of velogenic Newcastle disease virus (sub-genotype VII.1.1) from wild birds, with assessment of its pathogenicity in susceptible chickens. Animals. 11: 505 .
Sehrawat, R., Sharmam R., Ahlawat, S., Sharma, V., Thakur, M.S., Kaur, M., Tantia, M.S. (2021). First report on better functional property of black chicken meat from India. Indian Journal of Animal Research. 55: 727-733.

Śliżewska, K., Cukrowska, B., Smulikowska, S., Cielecka-Kuszyk, J. (2019). The effect of probiotic supplementation on performance and the histopathological changes in liver and kidneys in broiler chickens fed diets with aflatoxin B1. Toxins. 11: 112. 